\title{
Power Series Extender Method for the Solution of Nonlinear Differential Equations
}

\author{
Hector Vazquez-Leal ${ }^{1}$ and Arturo Sarmiento-Reyes ${ }^{2}$ \\ ${ }^{1}$ Electronic Instrumentation and Atmospheric Sciences School, Universidad Veracruzana, Cto. Gonzalo Aguirre Beltrán, \\ S/N, 91000 Xalapa, VER, Mexico \\ ${ }^{2}$ National Institute for Astrophysics, Optics and Electronics, Luis Enrique Erro No. 1, Santa Maria, 72840 Tonantzintla, PUE, Mexico \\ Correspondence should be addressed to Hector Vazquez-Leal; hvazquez@uv.mx
}

Received 1 October 2014; Accepted 1 December 2014

Academic Editor: Salvatore Alfonzetti

Copyright (c) 2015 H. Vazquez-Leal and A. Sarmiento-Reyes. This is an open access article distributed under the Creative Commons Attribution License, which permits unrestricted use, distribution, and reproduction in any medium, provided the original work is properly cited.

\begin{abstract}
We propose a power series extender method to obtain approximate solutions of nonlinear differential equations. In order to assess the benefits of this proposal, three nonlinear problems of different kind are solved and compared against the power series solution obtained using an approximative method. The problems are homogeneous Lane-Emden equation of $\alpha$ index, governing equation of a burning iron particle, and an explicit differential-algebraic equation related to battery model simulations. The results show that PSEM generates highly accurate handy approximations requiring only a few steps. The main advantage of PSEM is to extend the domain of convergence of the power series solutions of approximative methods as Taylor series method, homotopy perturbation method, homotopy analysis method, variational iteration method, differential transform method, and Adomian decomposition method, among many others. From the application of PSEM, it results in handy easy computable expressions that extend the domain of convergence of high order power series solutions.
\end{abstract}

\section{Introduction}

Solving nonlinear differential equations is an important task in sciences because many physical phenomena are modelled using such equations. Recently, the generalized homotopy method (GHM) [1] was proposed as a generalization of the homotopy perturbation method (HPM). The application of such method is based on a power series matching that enables GHM to obtain complex and rich expression impossible to obtain using HPM. Therefore, using as a guide the main idea of power series matching, behind GHM, we propose a power series method extender (PSEM). The key steps of this proposal are as follows.

(1) First, we apply an approximative method to obtain the coefficients of a power series solution. The approximative method can be one from the literature like Taylor series method (TSM) [2, 3], power series method (PSM) [4], homotopy perturbation method (HPM) [5-11], perturbation method (PM), homotopy analysis method (HAM), variational iteration method (VIM), differential transform method (DTM), and Adomian decomposition method (ADM), among many others.

(2) In the same fashion as GHM [1], we propose a trial function (TF) that can potentially describe the qualitative behaviour of the nonlinear problem.

(3) Next, we apply the Taylor series method to a trial function (TF).

(4) Then, we equate the coefficients of the power series obtained in steps 1 and 3 to obtain a nonlinear algebraic equation system in terms of the coefficients of the TF, which can be solved using symbolic or numerical methods.

(5) Finally, the approximate solution is obtained by substituting the calculated coefficients from step 4 into the proposed TF. 
In order to study the potential of the proposed technique, three nonlinear problems will be solved and compared versus numerical methods: homogeneous Lane-Emden equation of $\alpha$ index [12], governing equation of burning iron particle [13], and an explicit differential-algebraic equation related to battery model simulations [14].

This paper is organized as follows. In Section 2, we introduce the basic concept of PSEM method. In Section 3, we show the approximation of three nonlinear differential equations related to different phenomenons in physics. Numerical simulations and a discussion about the results are provided in Section 4. Finally, a brief conclusion is given in Section 5.

\section{Basic Concept of PSEM Method}

In a broad sense a nonlinear differential equation can be expressed as

$$
L(u)+N(u)-f(x)=0, \quad x \in \Omega,
$$

having as boundary condition

$$
B\left(u, \frac{\partial u}{\partial \eta}\right)=0, \quad x \in \Gamma,
$$

where $L$ and $N$ are a linear operator and a nonlinear operator, respectively, $f(x)$ is a known analytic function, $B$ is a boundary operator, $\Gamma$ is the boundary of domain $\Omega$, and $\partial u / \partial \eta$ denotes differentiation along the normal drawn outwards from $\Omega$ [8].

Next, we express the solution of (1) as a power series

$$
u=\sum_{k=0}^{\infty} v_{k} x^{k}
$$

where $v_{k}(k=0,1, \ldots)$ are the coefficients of the power series.

It is important to notice that (3) can be obtained by some approximative method from literature as HPM, HAM, VIM, DTM, ADM, TSM, and PSM, among others.

Now, we propose that the solution for (1) can be written as a finite sum of functions in the general form [1]

$$
u=u_{0}+\sum_{i=0}^{n} f_{i}\left(x, u_{i}\right),
$$

or

$$
u=\frac{u_{0}+\sum_{i=0}^{n} f_{i}\left(x, u_{i}\right)}{1+\sum_{j=n+1}^{2 n} f_{j}\left(x, u_{j}\right)},
$$

where $u_{i}$ are constants to be determined by PSEM, $f_{i}\left(x, u_{i}\right)$ are arbitrary trial functions, and $n$ and $2 n$ are the orders of approximations (4) and (5), respectively. We will denominate (4) and (5) as the trial function (TF).

Next, we calculate the Taylor series of (4) or (5), resulting in the power series

$$
\begin{aligned}
& u=u_{0}+\sum_{i=0}^{n} P_{i, 0}+\sum_{i=0}^{n} \sum_{k=1}^{\infty} P_{i, k} x^{k}, \\
& u=u_{0}+\sum_{i=0}^{n} P_{i, 0}+\sum_{i=0}^{2 n} \sum_{k=1}^{\infty} P_{i, k} x^{k},
\end{aligned}
$$

respectively, where Taylor coefficients $P_{k}$ are expressed in terms of parameters $u_{i}$.

Finally, we equate/match the coefficients of power series (6) or (7) with (3) to obtain the values of $u_{i}$ and substitute them into (4) or (5) to obtain the PSEM approximation.

It is important to notice that we can separately apply (4) or (5) to obtain an approximation of (1), where the selection of the TF depends of the behaviour of the problem under study. In addition, it is important to remark that if we choose the $f_{i}$ functions to be analytic, then (6) and (7) are convergent series [15-17].

\section{Case Studies}

In the present section, we will solve three case studies to show the utility of the PSEM method to solve nonlinear differential equations. We know from literature that approximative methods as HPM, PM, HAM (using the $h=-1$ as convergence control), VIM, DTM, ADM, TSM, and PSM are able to generate power series approximate solutions that in most of the cases are equivalent to the well-known TSM/PSM solutions. Therefore, the main difference in such cases is the difficulty of application of the specific approximative method to the particular case study. For its simplicity of application, we will use TSM [2] to exemplify the application of PSEM, although it is possible to use one of the available approximative methods from literature.

3.1. Homogeneous Lane-Emden Equation of $\alpha$ Index. The Lane-Emden singular equation describes a wide variety of problems in physics as some aspects related to the stellar structure, thermal history of spherical cloud of gas, isothermal gas spheres, and thermionic currents, among others [12]. The equation is expressed as

$$
\begin{aligned}
& y^{\prime \prime}+\frac{2}{x} y^{\prime}+y^{\alpha}=0, \quad 0<x \leq 1,0 \leq \alpha \leq 5 \\
& y(0)=1, \quad y^{\prime}(0)=0,
\end{aligned}
$$

where prime denotes differentiation with respect to $x$ and $\alpha$ represents the index of the equation.

Using the initial conditions of (8) and considering as expansion point $x_{0}=0$, it results the solution of (8) as

$$
\begin{aligned}
y(x)= & y(0)+\frac{y^{\prime}(0)}{1 !} x^{1}+\frac{y^{\prime \prime}(0)}{2 !} x^{2}+\frac{y^{\prime \prime \prime}(0)}{3 !} x^{3} \\
& +\frac{y^{(4)}(0)}{4 !} x^{4}+\cdots,
\end{aligned}
$$

where $y^{(m)}(0)(m=2,3, \ldots)$ are unknown constants to be determined by the Taylor series method.

In order to apply the TSM method [3], it is necessary to multiply (8) by $x$ to avoid the singularity, resulting in

$$
x y^{\prime \prime}+2 y^{\prime}+x y^{\alpha}=0 \text {. }
$$


Next, we derive successively (10) and resolve the system of equations obtained from the derivatives $y^{(m)}(0)(m=$ $2,3, \ldots)$, resulting in

$$
\begin{aligned}
& y^{\prime \prime}=\left(\frac{1}{3}\right)(\left.\left(-y-x \alpha y^{\prime}\right) y^{\alpha-1}-y^{\prime \prime \prime} x\right), \\
& y^{\prime \prime \prime}=\left(\frac{1}{4}\right)(-\alpha\left(x(\alpha-1) y^{\prime}+2 y\right) y^{\prime} y^{\alpha-2} \\
&\left.-y^{(\alpha-1)} y^{\prime \prime} \alpha x-y^{(4)} x\right), \\
& y^{(4)}=\left(\frac{1}{5}\right)\left(-3 y^{(\alpha-2)} y^{\prime \prime}\left(x(\alpha-1) y^{\prime}+y\right) \alpha\right. \\
&-(\alpha-1) \alpha\left(x(\alpha-2) y^{\prime}+3 y\right) y^{\prime 2} y^{\alpha-3} \\
&\left.-y^{(\alpha-1)} \alpha x y^{\prime \prime \prime}-y^{(5)} x\right),
\end{aligned}
$$

From (5), we propose a specific solution for (8) with the following form:

$$
y(x)=\frac{y_{0}+y_{1} x^{1}+y_{2} x^{2}+\cdots+y_{n} x^{n}}{1+y_{n+1} x^{1}+y_{n+2} x^{2}+\cdots+y_{2 n} x^{n}},
$$

where $y_{i}(i=0,1,2, \ldots)$ are constants to be determined and the order $n$ is chosen as 2 to obtain a handy approximation.

Next, Taylor series of (13) is

$$
\begin{aligned}
y(x)= & y_{0}+\left(y_{1}-y_{0} y_{3}\right) x \\
+ & \left(y_{2}-y_{0} y_{4}+\left(-y_{1}+y_{0} y_{3}\right) y_{3}\right) x^{2} \\
+ & \left(\left(-y_{1}+y_{0} y_{3}\right) y_{4}\right. \\
& \left.+\left(-y_{2}+y_{0} y_{4}+y_{3} y_{1}-y_{0} y_{3}^{2}\right) y_{3}\right) x^{3} \\
+ & \left(\left(-y_{2}+y_{0} y_{4}+y_{3} y_{1}-y_{0} y_{3}^{2}\right) y_{4}\right. \\
& +\left(y_{4} y_{1}-2 y_{4} y_{0} y_{3}+y_{3} y_{2}-y_{3}^{2} y_{1}\right. \\
& \left.\left.+y_{0} y_{3}^{3}\right) y_{3}\right) x^{4}+\cdots .
\end{aligned}
$$

Then, we equate coefficients of $x$-powers of (9) and (14) to obtain a system of equations, which can be solved symbolically, resulting in

$$
\begin{aligned}
& y_{0}=1, \\
& y_{1}=0, \\
& y_{2}=\frac{\alpha}{20}-\frac{1}{6}, \\
& y_{3}=0, \\
& y_{4}=\frac{\alpha}{20} .
\end{aligned}
$$

Finally, the PSEM approximation is obtained by substituting (15) into (13), resulting in

$$
y(x)=\frac{1+(\alpha / 20-1 / 6) x^{2}}{1+(\alpha / 20) x^{2}}, \quad 0<x \leq 1,0 \leq \alpha \leq 5 .
$$

3.2. Combustion of a Single Iron Particle. The nonlinear energy equation for combustion of a single iron particle [13] is

$$
\begin{array}{r}
\left(1-\epsilon_{1}\left(\theta-\theta_{\infty}\right)\right) \theta^{\prime}+\theta-\theta_{\infty}+\epsilon_{2}\left(\theta^{4}-\theta_{\text {surr }}^{4}\right)-\Psi=0, \\
\theta(0)=1,
\end{array}
$$

where prime denotes differentiation with respect to $\tau$ and $\theta$ is the dimensionless temperature.

The values of the parameters are obtained from [13]: $\theta_{\infty}=$ $1.17647, \epsilon_{1}=0.051595, \Psi=0.98579, \theta_{\text {surr }}=0.35294$, and $\epsilon_{2}=0.002630145546$. Using the initial conditions of (17) and considering the expansion point $\tau=0$ yields to the following Taylor series:

$$
\theta(\tau)=\theta(0)+\frac{\theta^{\prime}(0)}{1 !} \tau^{1}+\frac{\theta^{\prime \prime}(0)}{2 !} \tau^{2}+\cdots,
$$

where $\theta^{(m)}(0)(m=1,2, \ldots)$ are unknown constants.

Now, we resolve (17) for $\theta^{\prime}$ and calculate the successive derivatives to obtain $\theta^{(m)}(0)(m=1,2, \ldots)$, resulting in

$$
\begin{aligned}
\theta^{\prime}= & -\frac{\theta-\theta_{\infty}+\epsilon_{2}\left(\theta^{4}-\theta_{\text {surr }}^{4}\right)-\Psi}{1-\epsilon_{1}\left(\theta-\theta_{\infty}\right)}, \\
\theta^{\prime \prime}= & -\frac{\epsilon_{1} \theta^{\prime}\left(\theta-\theta_{\infty}+\epsilon_{2}\left(\theta^{4}-\theta_{\text {surr }}^{4}\right)-\Psi\right)}{\left(1-\epsilon_{1}\left(\theta-\theta_{\infty}\right)\right)^{2}} \\
& -\frac{\theta^{\prime}+4 \epsilon_{2} \theta^{3} \theta^{\prime}}{1-\epsilon_{1}\left(\theta-\theta_{\infty}\right)}
\end{aligned}
$$


Then, substituting the initial conditions of (17) into (19), we get

$$
\begin{aligned}
\theta^{\prime}(0)= & -\frac{\theta(0)-\theta_{\infty}+\epsilon_{2}\left(\theta(0)^{4}-\theta_{\text {surr }}^{4}\right)-\Psi}{1-\epsilon_{1}\left(\theta(0)-\theta_{\infty}\right)} \\
\theta^{\prime \prime}(0)= & -\frac{\epsilon_{1} \theta^{\prime}(0)\left(\theta(0)-\theta_{\infty}+\epsilon_{2}\left(\theta(0)^{4}-\theta_{\text {surr }}^{4}\right)-\Psi\right)}{\left(1-\epsilon_{1}\left(\theta(0)-\theta_{\infty}\right)\right)^{2}} \\
& -\frac{\theta^{\prime}(0)+4 \epsilon_{2} \theta(0)^{3} \theta^{\prime}(0)}{1-\epsilon_{1}\left(\theta(0)-\theta_{\infty}\right)}
\end{aligned}
$$

From (4), we propose a specific solution for (17) as follows:

$$
\theta(\tau)=\theta_{0}+\theta_{1} \exp \left(\theta_{2} \tau\right)
$$

Next, Taylor series of (21) is

$$
\theta(\tau)=\theta_{0}+\theta_{1}+\theta_{1} \theta_{2} \tau+\left(\frac{1}{2}\right) \theta_{1} \theta_{2}^{2} \tau^{2}+\cdots
$$

Then, we equate coefficients of $\tau$-powers of (18) and (22) to obtain a system of equations, which can be solved symbolically, resulting in

$$
\begin{aligned}
& \theta_{0}=\frac{\left(k_{0} k_{2}-k_{1}^{2}\right)}{k_{2}}, \\
& \theta_{1}=\frac{k_{1}^{2}}{k_{2}} \\
& \theta_{2}=\frac{k_{2}}{k_{1}}
\end{aligned}
$$

where $k_{0}=\theta(0), k_{1}=\theta^{\prime}(0)$, and $k_{2}=\theta^{\prime \prime}(0)$.

Finally, substituting (23) into (21) yields to the PSEM approximation

$$
\theta(\tau)=\frac{\left(k_{0} k_{2}-k_{1}^{2}\right)}{k_{2}}+\left(\frac{k_{1}^{2}}{k_{2}}\right) \exp \left(\frac{k_{2} \tau}{k_{1}}\right) .
$$

For comparison purposes, we calculate the Taylor series by substituting $\theta(0)=1$ and (20) into (18), yielding

$$
\theta(\tau)=1+1.170326453 \tau-0.6324114785 \tau^{2} .
$$

3.3. Differential-Algebraic Equation Related to Battery Model Simulation. Next, PSEM will be applied to a nonlinear equation [14] related to an oversimplified battery model, which is formulated as follows:

$$
\begin{gathered}
z^{\prime}=-2 z+y^{2}, \quad z(0)=2, \\
-100 \ln (y)+2 z=5, \quad y(0)=\exp (-0.01),
\end{gathered}
$$

where $z$ is the differential variable, $y$ is the algebraic variable, and primes denote derivative with respect to $t$.

Using the initial conditions of (26) and considering the expansion point $t=0$ yields to the following Taylor series:

$$
\begin{aligned}
z(t)= & z(0)+\frac{z^{\prime}(0)}{1 !} t^{1}+\frac{z^{\prime \prime}(0)}{2 !} t^{2}+\frac{z^{\prime \prime \prime}(0)}{3 !} t^{3} \\
& +\frac{z^{(4)}(0)}{4 !} t^{4}+\cdots, \\
y(t)= & y(0)+\frac{y^{\prime}(0)}{1 !} t^{1}+\frac{y^{\prime \prime}(0)}{2 !} t^{2}+\frac{y^{\prime \prime \prime}(0)}{3 !} t^{3}
\end{aligned}
$$

$$
+\frac{y^{(4)}(0)}{4 !} t^{4}+\cdots
$$

where derivatives $z^{(m)}(0)$ and $y^{(m)}(0)(m=1,2, \ldots)$ are unknown.

Firstly, we apply an implicit derivative with respect to $t$ of the second equation of (26), resulting in

$$
\begin{gathered}
z^{\prime}=-2 z+y^{2}, \quad z(0)=2 \\
y^{\prime}=\left(\frac{1}{50}\right) z^{\prime} y, \quad y(0)=\exp (-0.01) .
\end{gathered}
$$

As before, we calculate the successive derivatives of (28) and evaluate the resulting equations at $t=0$, yielding

$$
\begin{aligned}
& z(0)=\gamma, \\
& z^{\prime}(0)=\zeta^{2}-2 \gamma, \\
& z^{\prime \prime}(0)=\left(\frac{1}{25}\right)\left(\zeta^{2}-50\right)\left(\zeta^{2}-2 \gamma\right), \\
& z^{\prime \prime \prime}(0)=\frac{2}{625}\left(\zeta^{2}-2 \gamma\right)\left(\zeta^{4}+1250+(-\gamma-50) \zeta^{2}\right), \\
& z^{(4)}(0)=\frac{6}{15625}\left(-\frac{62500}{3}+\zeta^{6}+\left(-2 \gamma-\frac{175}{3}\right) \zeta^{4}\right. \\
& \quad \vdots \\
& \left.y^{(0)}=\zeta\left(\frac{200}{3} \gamma+1250+\left(\frac{2}{3}\right) \gamma^{2}\right) \zeta^{2}\right) \\
& y^{\prime}(0)=\frac{1}{50}\left(\zeta^{2}-2 \gamma\right)(\zeta), \\
& y^{\prime \prime}(0)=\frac{3}{2500}(\zeta)\left(\zeta^{2}-2 \gamma\right)\left(\zeta^{2}-\frac{2}{3} \gamma-\frac{100}{3}\right),
\end{aligned}
$$




$$
\begin{array}{r}
y^{\prime \prime \prime}(0)=\frac{3}{25000}(\zeta)\left(\zeta^{2}-2 \gamma\right)\left(\zeta^{4}+\left(-\frac{140}{3}-\frac{8}{5} \gamma\right) \zeta^{2}\right. \\
\left.+40 \gamma+\frac{2000}{3}+\frac{4}{15} \gamma^{2}\right) \\
y^{(4)}(0)=\frac{21}{1250000}(\zeta)\left(\zeta^{6}+\left(-\frac{1240}{21}-\frac{18}{7} \gamma\right) \zeta^{4}\right. \\
+\left(\frac{26000}{21}+\frac{320}{3} \gamma+\frac{52}{35} \gamma^{2}\right) \zeta^{2} \\
-\frac{4000}{3} \gamma-\frac{8}{105} \gamma^{3} \\
\left.\quad-\frac{160}{7} \gamma^{2}-\frac{200000}{21}\right) \\
\times\left(\zeta^{2}-2 \gamma\right) \quad
\end{array}
$$

where $\gamma=z(0)$ and $\zeta=y(0)$.

From (4), we propose a specific solution for (26) as follows:

$$
\begin{aligned}
& z(t)=z_{0}+z_{1} \exp \left(z_{2} t\right)+z_{3} \exp \left(z_{4} t\right), \\
& y(t)=y_{0}+y_{1} \exp \left(y_{2} t\right)+y_{3} \exp \left(y_{4} t\right) .
\end{aligned}
$$

Finally, we calculate the fourth-order Taylor series of (30), equate the resulting coefficients of the same $t$-powers with respect to (27), and solve the two systems of equations, resulting in the following PSEM approximation:

$$
\begin{aligned}
z(t)= & 0.4608270703+1.539992595 \exp (-1.963069736 t) \\
& -0.0008196648996 \exp (-4.040101111 t), \\
y(t)= & 0.9600410114+0.02960275048 \exp (-1.964621532 t) \\
& +0.0004060718378 \exp (-4.030998609 t) .
\end{aligned}
$$

For comparison purposes, we calculate the Taylor series by substituting (29) into (27), yielding

$$
\begin{aligned}
z(t)= & 2-3.019801327 t+2.960601222 t^{2}-1.932657483 t^{3} \\
& +0.9438075192 t^{4} \\
y(t)= & 0.9900498337-0.05979507603 t+0.06042854745 t^{2} \\
& -0.04184548412 t^{3}+0.02284265412 t^{4}
\end{aligned}
$$

\section{Numerical Simulation and Discussion}

For all case studies, we used built-in numerical routines from Maple 15 for comparison purposes. The Fehlberg fourthfifth order Runge-Kutta method with degree four interpolant (RKF45) was used $[18,19]$. The command was setup with a tolerance of absolute error (A. E.) of $10^{-12}$.
We obtained a highly accurate rational approximate solution (16) for the singular second order Lane-Emden equation [12] (8) as depicted in Figures 1 and 2 for $\alpha=$ $[1,2,3,4,5]$. Thus, the PSEM method may be useful for such kind of problems with singularities.

Additionally, we solved the nonlinear energy equation for combustion of a single iron particle [13] (17) obtaining a highly accurate approximation (24) as depicted in Figures 3 and 4 . In the same figures, we can observe the Taylor series solution (25), noticing the higher precision of the proposed solution.

Finally, we approximated a simplistic model [14] related to a battery model simulation that is represented as a nonlinear differential-algebraic equation of index 1 (26). The PSEM approximation (31) is in good agreement to numerical results (RKF45) for a large period of time, as depicted in Figure 5; in contrast, we can observe in the same figure the poor convergence of the Taylor series solution. As a matter of fact, if we calculate the asymptotic behaviour of (26), we will conclude that the approximation keeps its high accuracy for a very large period of time. On one side, setting the $z^{\prime}(t)=0$ in (26) and solving the system, it results in that the stable equilibrium point is $E_{1}=\left[z_{\infty}=0.4608356746, y_{\infty}=\right.$ $0.9600371604]$. On the other side, we calculate the limit when $t \rightarrow \infty$ of (31), resulting in $E_{2}=\left[z_{\infty}=0.4608270703, y_{\infty}=\right.$ $0.9600410114]$. Therefore, the high proximity among $E_{1}$ and $E_{2}$ exhibits the high accuracy of the proposed PSEM approximation for the rank $t \in[0, \infty)$.

The PSEM method was able to calculate approximations with a larger domain of convergence in comparison to the TSM results. The reason can lie in the fact that the trial function of PSEM technique may potentially contain more information than the Taylor power series. As long as the Taylor series of the proposed TF exist, we can propose a wide variety of series of functions as: trigonometric, hyperbolic, integrals, among many others. However, further research is required to propose a methodology to choose the optimal TF. At the moment, users of the method should keep in mind proposing a TF that may potentially reproduce the behaviour of the exact solution. Finally, it is important to remark that as the $\mathrm{TF}$ is a finite sum or division of analytic functions, as shown in (4) or (5), therefore such sum is convergent [15-17]; additionally, we will require only a finite number of terms of the Taylor expansions (6) or (7) to obtain the coefficients of the trial functions (4) or (5). Finally, it is important to remark that PSEM can be easily combined with HPM, PM, HAM, VIM, DTM, and ADM, among many others. Furthermore, we can select the approximative method depending on its facility of application to the specific case study. Therefore, PSEM is a powerful malleable technique that can be applied in combination with many of the approximative methods reported in literature.

\section{Conclusions}

This work introduced PSEM as a useful tool with high potential to solve nonlinear differential equations. We were 


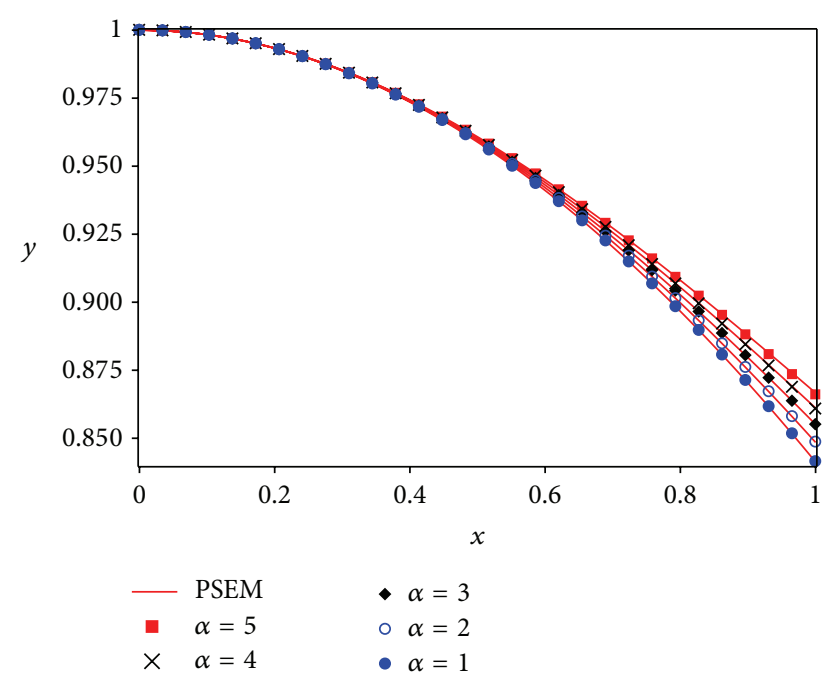

FIGURE 1: RKF45 solution for (8) (symbols) and its approximate PSEM solution (16) (solid line) for different $\alpha$ values.

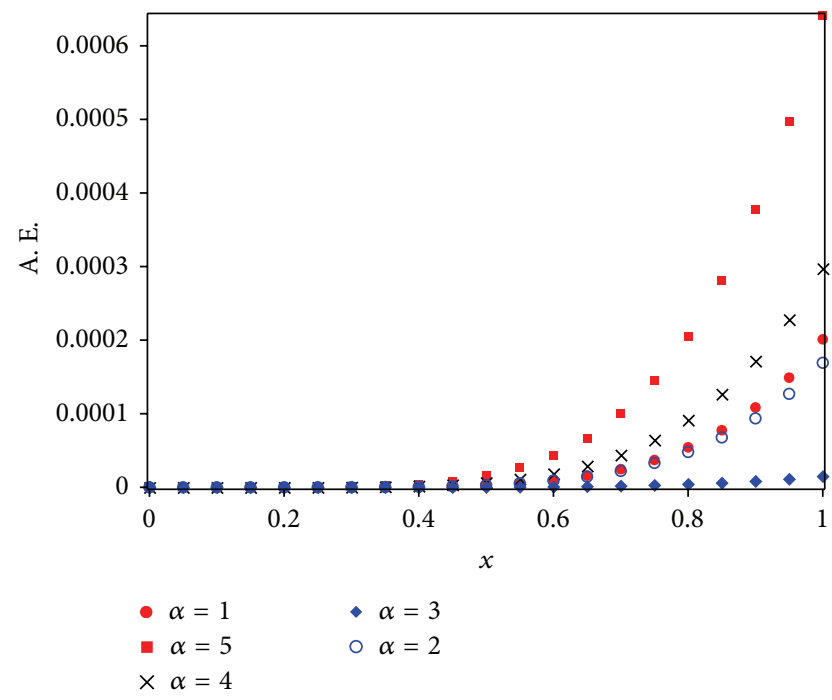

Figure 2: Absolute error (A. E.) of (16) with respect to numerical (RKF45) solution for (8).

able to obtain accurate and handy approximations for different types of problems: homogeneous singular Lane-Emden equation, nonlinear energy equation for combustion of a single iron particle, and differential-algebraic system related to battery model simulation. It is important to remark on the high flexibility, applicability, and power of this novel method. A key point that should be remarked is that the user should propose a finite sum of division of analytic functions (trial function) that may be chosen according to the nature of the nonlinear problem under study. Further research is required to solve other kinds of problems: nonlinear fractional differential equations, nonlinear partial differential equations, and nonlinear boundary valued problems, among others.

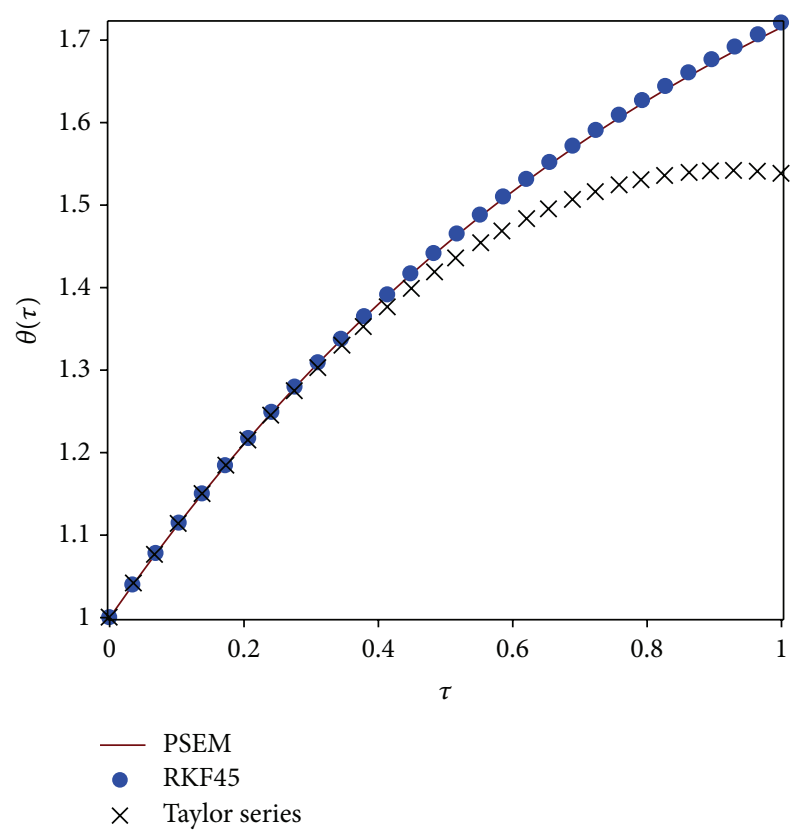

FIGURE 3: RKF45 solution for (17) (solid circles), its approximate PSEM solution (24) (solid line), and its Taylor series solution (25) (diagonal crosses).

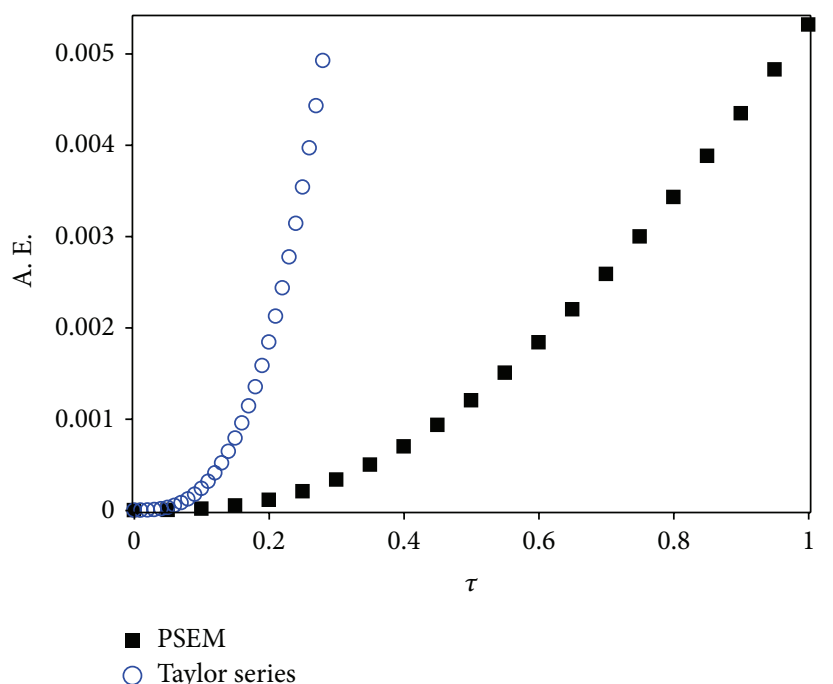

FIgURE 4: Absolute error (A. E.) of (24) (solid square) and (25) (empty circle) with respect to numerical (RKF45) solution for (17).

\section{Conflict of Interests}

The authors declare that there is no conflict of interests regarding the publication of this paper.

\section{Acknowledgments}

The authors gratefully acknowledge the financial support from the National Council for Science and Technology of Mexico (CONACyT) through Grant CB-2010-01 no. 


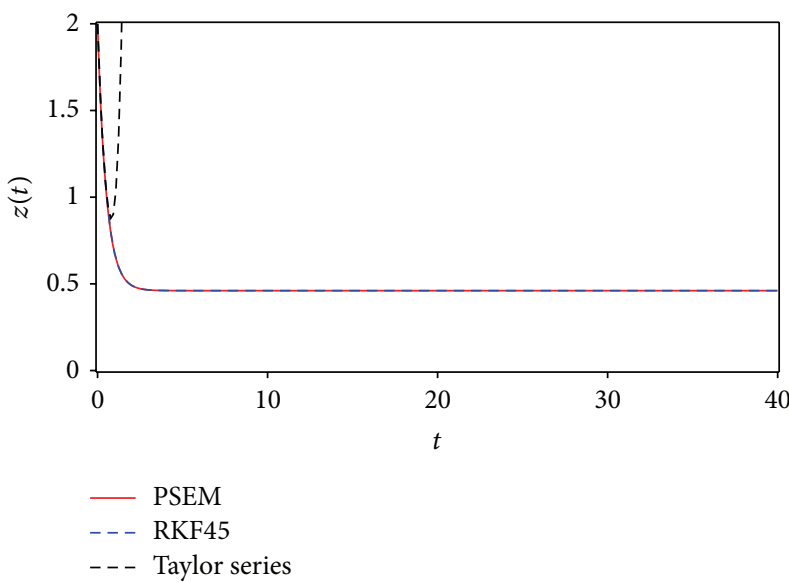

(a)

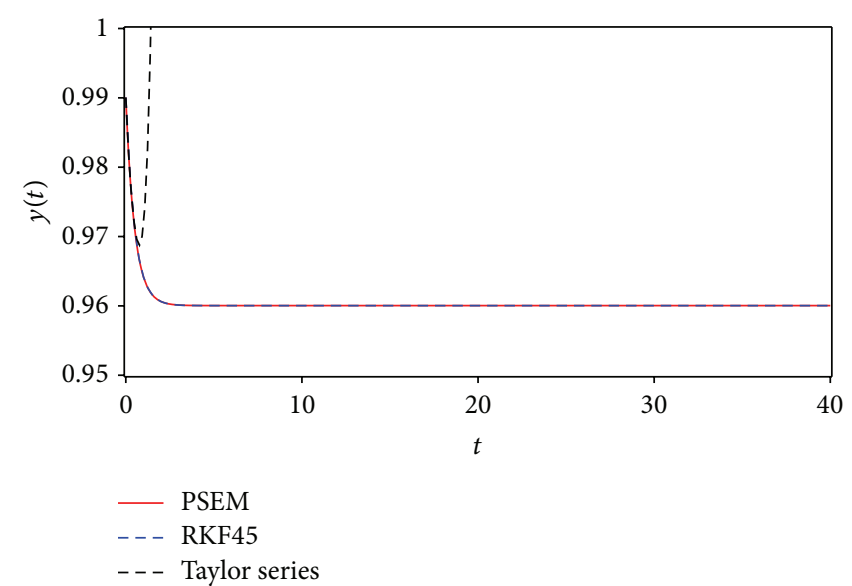

(b)

FIGURE 5: RKF45 solution for (26) (solid circles), its approximate PSEM solution (31) (solid line), and its Taylor series solution (32) (solid square).

157024. The authors would like to thank Roberto CastanedaSheissa, Uriel Filobello-Nino, Rogelio-Alejandro CallejasMolina, and Roberto Ruiz-Gomez for their contribution to this project.

\section{References}

[1] H. Vazquez-Leal, "Generalized homotopy method for solving nonlinear differential equations," Computational and Applied Mathematics, vol. 33, no. 1, pp. 275-288, 2014.

[2] H. Vazquez-Leal, B. Benhammouda, U. A. Filobello-Nino et al., "Modified Taylor series method for solving nonlinear differential equations with mixed boundary conditions defined on finite intervals," SpringerPlus, vol. 3, no. 1, article 160, 2014.

[3] R. Barrio, M. Rodríguez, A. Abad, and F. Blesa, "Breaking the limits: the Taylor series method," Applied Mathematics and Computation, vol. 217, no. 20, pp. 7940-7954, 2011.

[4] H. Vazquez-Leal, "Exact solutions for differential-algebraic equations," Miskolc Mathematical Notes, vol. 15, no. 1, pp. 227238,2014

[5] J.-H. He, "Homotopy perturbation technique," Computer Methods in Applied Mechanics and Engineering, vol. 178, no. 3-4, pp. 257-262, 1999.

[6] A. Barari, M. Omidvar, A. R. Ghotbi, and D. D. Ganji, "Application of homotopy perturbation method and variational iteration method to nonlinear oscillator differential equations," Acta Applicandae Mathematicae, vol. 104, no. 2, pp. 161-171, 2008.

[7] Y. Khan, H. Vázquez-Leal, and Q. Wu, "An efficient iterated method for mathematical biology model," Neural Computing and Applications, vol. 23, no. 3-4, pp. 677-682, 2013.

[8] Y.-G. Wang, W.-H. Lin, and N. Liu, "A homotopy perturbationbased method for large deflection of a cantilever beam under a terminal follower force," International Journal for Computational Methods in Engineering Science and Mechanics, vol. 13, no. 3, pp. 197-201, 2012.

[9] M. A. Fariborzi Araghi and B. Rezapour, "Application of homotopy perturbationmethod to solve multidimensional Schrödinger's equations," International Journal of Mathematical Archive, vol. 2, no. 11, pp. 1-6, 2011.
[10] M. Bayat, I. Pakar, and A. Emadi, "Vibration of electrostatically actuated microbeam by means of homotopy perturbation method," Structural Engineering and Mechanics, vol. 48, no. 6, pp. 823-831, 2013.

[11] M. M. Rashidi, M. T. Rastegari, M. Asadi, and O. A. Bég, "A study of non-Newtonian flow and heat transfer over a non-isothermal wedge using the homotopy analysis method," Chemical Engineering Communications, vol. 199, no. 2, pp. 231256, 2012.

[12] A. Akyüz-Daşcioğlu and H. Cerdiůk-Yaslan, "The solution of high-order nonlinear ordinary differential equations by Chebyshev series," Applied Mathematics and Computation, vol. 217, no. 12, pp. 5658-5666, 2011.

[13] M. Bidabadi and M. Mafi, "Time variation of combustion temperature and burning time of a single iron particle," International Journal of Thermal Sciences, vol. 65, pp. 136-147, 2013.

[14] R. N. Methekar, V. Ramadesigan, J. C. Pirkle Jr., and V. R. Subramanian, "A perturbation approach for consistent initialization of index-1 explicit differential-algebraic equations arising from battery model simulations," Computers \& Chemical Engineering, vol. 35, no. 11, pp. 2227-2234, 2011.

[15] D. G. Zill, A First Course in Differential Equations with Modeling Applications, Cengage Learning, Boston, Mass, USA, 10th edition, 2012.

[16] W. Belser, Formal Power Series and Linear Systems of Meromorphic Ordinary Differential Equations, Springer, 1999.

[17] M. Oberguggenberger and A. Ostermann, Analysis for Computer Scientists: Foundations, Methods, and Algorithms, Springer, 2011.

[18] W. H. Enright, K. R. Jackson, S. P. Nørsett, and P. G. Thomsen, "Interpolants for Runge-Kutta formulas," ACM Transactions on Mathematical Software, vol. 12, no. 3, pp. 193-218, 1986.

[19] E. Fehlberg, "Klassische runge-kutta-formeln vier ter und niedrigerer ordnung mit schrittweitenkontrolle und ihre anwendung auf waermeleitungsprobleme," Computing, vol. 6, pp. 61-71, 1970. 


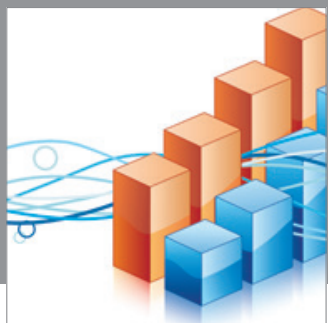

Advances in

Operations Research

mansans

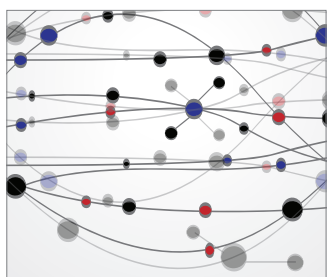

The Scientific World Journal
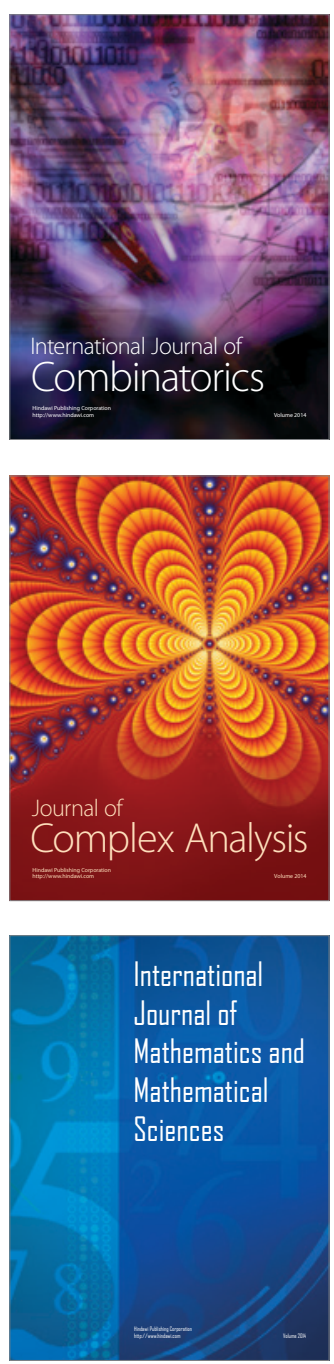
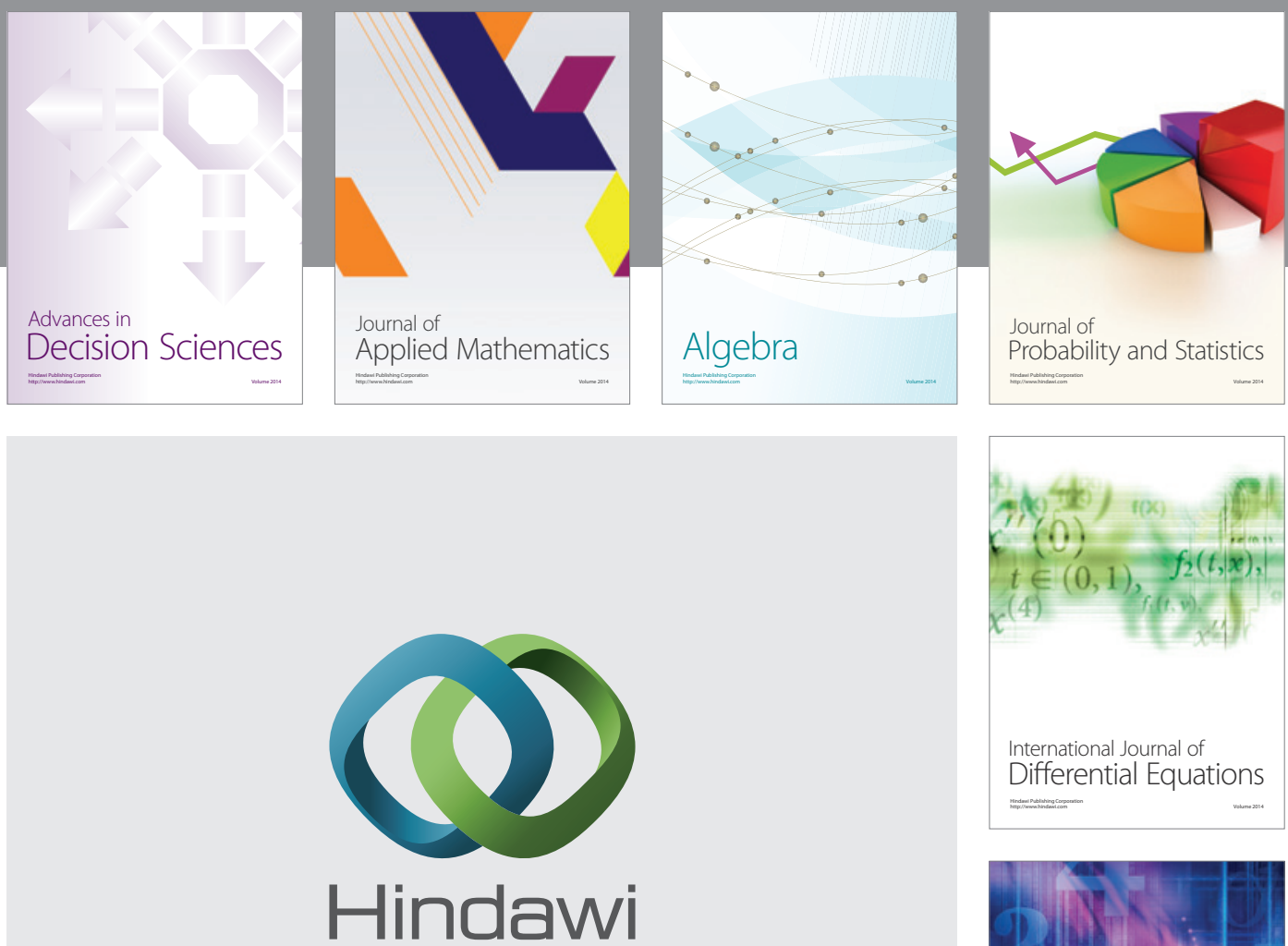

Submit your manuscripts at http://www.hindawi.com
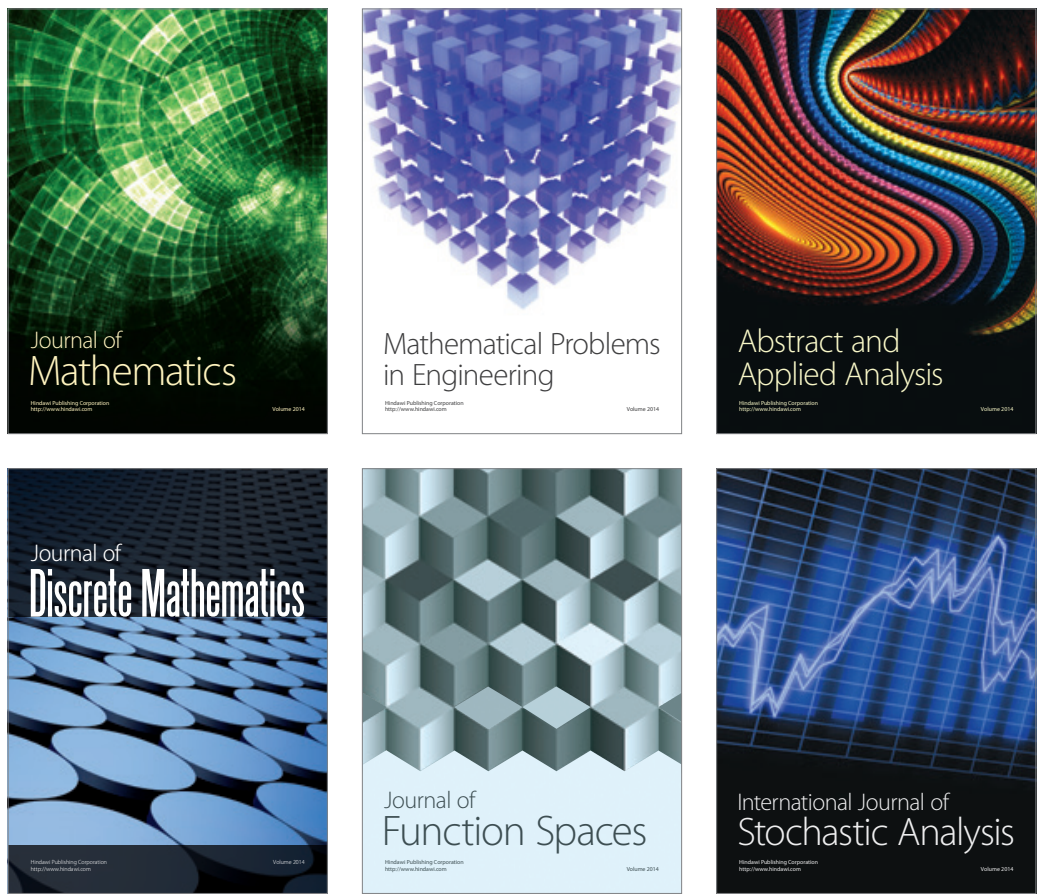

Journal of

Function Spaces

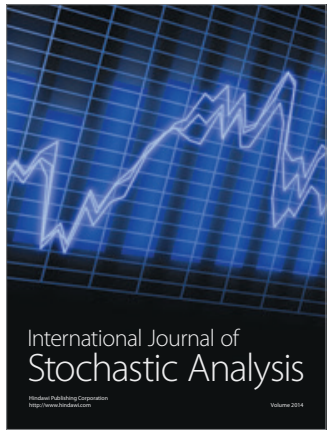

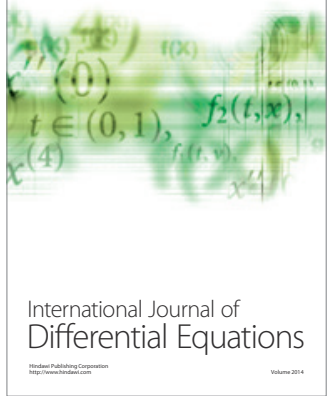
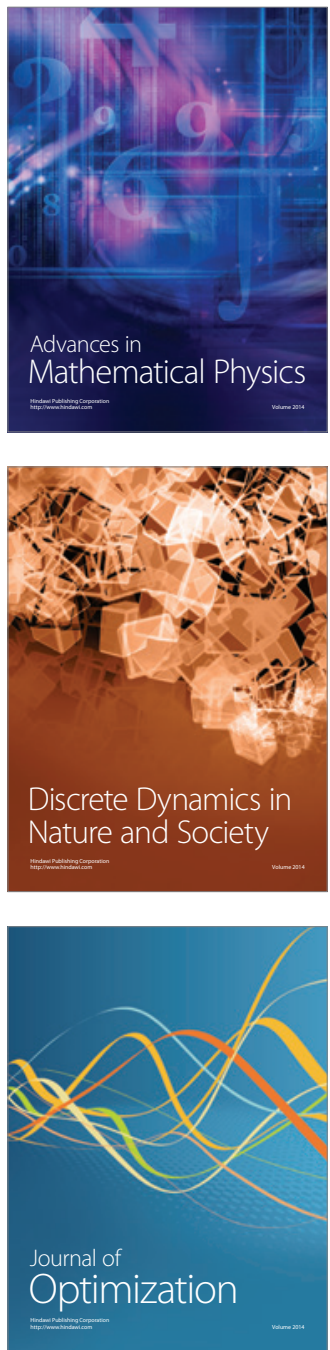